\title{
The Finite Element Method With Lagrange Multipliers for Domains With Corners*
}

\author{
By Juhani Pitkäranta
}

\begin{abstract}
We study the convergence of the finite element method with Lagrange multipliers for approximately solving the Dirichlet problem for a second-order elliptic equation in a plane domain with piecewise smooth boundary. Assuming mesh refinements around the corners, we construct families of boundary subspaces that are compatible with triangular Lagrange elements in the interior, and we carry out the error analysis of the resulting approximations in weighted Sobolev spaces.
\end{abstract}

1. Introduction. We consider the model problem

$$
\begin{aligned}
-\Delta u=f & \text { in } \Omega, \\
u=g & \text { on } \partial \Omega,
\end{aligned}
$$

where $\Omega \subset R^{2}$ is a bounded domain with piecewise smooth boundary $\partial \Omega$, and $f$ and $g$ are given functions defined on $\Omega$. Under sufficient regularity hypotheses on $f$ and $g$, problem (1.1) admits the following weak formulation: Find a pair $(u, \psi)$, $u \in H^{1}(\Omega), \psi \in L_{2}(\partial \Omega)$, such that

$$
\begin{aligned}
& \int_{\Omega} \sum_{i=1}^{2} \frac{\partial u}{\partial x_{i}} \frac{\partial v}{\partial x_{i}} d x+\int_{\partial \Omega} v \psi d x+\int_{\partial \Omega} u \phi d s \\
&=\int_{\Omega} f v d x+\int_{\partial \Omega} g \phi d s \quad \text { for all }(v, \phi) \in H^{1}(\Omega) \times L_{2}(\partial \Omega) .
\end{aligned}
$$

For $f, g$ smooth enough, $(1.2)$ has a unique solution $(u, \psi)$ such that $u$ is the weak solution of (1.1) and $\psi$ is defined as

$$
\psi=-\frac{\partial u}{\partial n}=-n_{1} \frac{\partial u}{\partial x_{1}}-n_{2} \frac{\partial u}{\partial x_{2}}, \quad x \in \partial \Omega,
$$

where $n(x)=\left(n_{1}(x), n_{2}(x)\right)$ denotes the unit outward normal vector of $\partial \Omega$ at $x$.

In the Lagrange multiplier method [2] for approximately solving (1.2) one introduces the finite-dimensional subspaces $M^{h} \subset H^{1}(\Omega)$ and $N^{h} \subset L_{2}(\partial \Omega)$ and defines the approximate solution as the pair $\left(u_{h}, \psi_{h}\right) \in M^{h} \times N^{h}$ which satisfies

$$
\begin{aligned}
\int_{\Omega} \sum_{i=1}^{2} \frac{\partial u_{h}}{\partial x_{i}} \frac{\partial v}{\partial x_{i}} d x & +\int_{\partial \Omega} v \psi_{h} d s+\int_{\partial \Omega} u_{h} \phi d s \\
= & \int_{\Omega} f v d x+\int_{\partial \Omega} g \phi d s \quad \text { for all }(v, \phi) \in M^{h} \times N^{h}
\end{aligned}
$$

Received September 6, 1979; revised July 9, 1980 and November 24, 1980.

1980 Mathematics Subject Classification. Primary 65N30.

- This work was supported by the Finnish National Research Council for Technical Sciences. 
Finite element methods based on (1.4) have the benefit that they do not require the fulfillment of the Dirichlet boundary condition in the subspaces. The stability and convergence of such approximations was studied first in [2] (see also [3]). For an abstract Lagrange multiplier method, necessary and sufficient conditions for stability are given in [6].

The actual verification of stability and the construction of the subspaces was considered by the author in [9] for quasi-uniform finite element meshes and in [10] in a more general situation. In [10] a number of local conditions were stated that yield the uniform stability of the Lagrange multiplier method in weighted norms depending on the finite element partitioning of the boundary.

In the present paper we proceed from the ideas of [10] and [4]. We consider the situation where graded meshes are used to handle the singularities arising from the corners of $\partial \Omega$. Assuming mesh refinements of the same type as those considered in [4] for polygonal domains, we construct finite element subspaces that meet with the stability conditions of [10]. We then derive error estimates for the resulting approximations in weighted Sobolev spaces.

The plan of the paper is as follows. In Section 2 we state the basic assumptions and give some properties of weighted Sobolev spaces that are needed in the paper. In Section 3 the stability conditions are restated from [10]. A family of finite element subspaces is introduced in Section 4 and the validity of the stability conditions is verified. Finally, in Section 5, the approximability properties of the subspaces in weighted Sobolev spaces are studied, and a weighted convergence result is derived for the approximate solution.

2. Preliminaries. We consider a bounded, simply connected domain $\Omega \subset R^{\mathbf{2}}$, whose boundary $\partial \Omega$ consists of a finite number of smooth closed arcs $\Gamma_{i}, i=$ $1, \ldots, I$, defined in terms of the smooth mappings $J_{i}=\left(J_{i, 1}, J_{i, 2}\right)$ such that

$$
\begin{aligned}
\Gamma_{i}=\left\{\left(J_{i, 1}(t), J_{i, 2}(t)\right), t\right. & \in[0,1]\}, \\
& {\left[J_{i, 1}^{\prime}(t)\right]^{2}+\left[J^{\prime}{ }_{1,2}(t)\right]^{2} \geqslant C>0, \quad t \in[0,1] . }
\end{aligned}
$$

We will denote the corners, i.e., the endpoints of the arcs $\Gamma_{i}$, by $z_{i}, i=1, \ldots, I$, numbered in any order. The asymptotic opening angles of the corners into the interior of $\Omega$ are denoted by $\omega_{i}$. We assume that $0<\omega_{i}<2 \pi, i=1, \ldots, I$, i.e., we exclude needles but include slits.

For $u(x)$ defined on $\Omega$, let $D^{k} u$ denote the field of all partial derivatives of $u$ of order $k$. Then the Sobolev space $H^{m}(\Omega), m>0$, is defined as usual, with the norm

$$
\|u\|_{H^{m(\Omega)}}^{2}=\sum_{k=0}^{m} \int_{\Omega}\left|D^{k} u\right|^{2} d x .
$$

In what follows, we use the notation

$$
\varphi_{\beta}(x)=\prod_{i=1}^{I}\left|x-z_{i}\right|^{\beta_{i}}, \quad \beta=\left(\beta_{1}, \ldots, \beta_{I}\right) \in R^{I},
$$

and $\theta=(1,1, \ldots, 1) \in R^{I}$. For $\beta \in R^{I}$ and $m>0$ given, we let $W^{m, \beta}(\Omega)$ denote the weighted Sobolev space of functions $u$, defined on $\Omega$, such that

$$
\|u\|_{W^{m, \beta}(\Omega)}^{2}=\sum_{k=0}^{m} \int_{\Omega} \varphi_{\beta+(k-m) \theta}^{2}\left|D^{k} u\right|^{2} d x<\infty .
$$


We state below some inequalities to be needed later on.

Lemma 2.1. If $\beta \in R^{I}$ is such that $0<\beta_{i}<1, i=1, \ldots, I$, and $u \in H^{1}(\Omega)$, then

$$
\int_{\Omega} \varphi_{\beta}^{-2} u^{2} d x<C\|u\|_{H^{1}(\Omega)}^{2}
$$

where $C$ depends on $\beta$ and $\Omega$.

Proof. Let $\chi_{i, \delta}$ be a smooth function defined on $\Omega$ such that $\chi_{i, \delta}(x) \equiv 1$ for $\left|x-z_{i}\right|<\delta$ and $\chi_{i, \delta}(x) \equiv 0$ for $\left|x-z_{i}\right|>2 \delta$. Then, if $\delta$ is sufficiently small, a modification of Hardy's inequality gives (see [8]):

$$
\int_{\Omega}\left|x-z_{i}\right|^{\alpha}\left(\chi_{i, \delta} u\right)^{2} d x \leqslant C \int_{\Omega}\left|x-z_{i}\right|^{\alpha+2}\left|D^{1}\left(\chi_{i, \delta} u\right)\right|^{2} d x, \quad \alpha>-2 .
$$

Taking $\alpha=-2 \beta_{i}$, summing over $i$ and using the triangle inequality, the assertion follows after a simple computation.

LEMMA 2.2. If $\beta \in R^{I}$ is such that $\beta_{i}<1$ for some $i \in\{1, \ldots, I\}$, then any $u \in W^{2, \beta}(\Omega)$ satisfies

$$
|u(x)| \leqslant C\|u\|_{W^{2, \beta}(\Omega)}, \quad\left|x-z_{j}\right|>\delta>0, j \neq i,
$$

where $C$ depends on $\Omega$ and $\delta$. Moreover, $u\left(z_{i}\right)=0$.

Proof. See [4]. This result is also implicit in [8].

Consider next functions defined on $\partial \Omega$. We say $\psi \in H^{m}(\partial \Omega), m \geqslant 0$, if

$$
\|\psi\|_{H^{m(\partial \Omega)}}^{2}=\sum_{i=1}^{I}\left\|\psi_{i}\right\|_{H^{m}(0,1)}^{2}<\infty
$$

where $\psi_{i}(t)=\psi\left(J_{i}(t)\right), t \in(0,1), i=1, \ldots, I$. Similarly, $\psi \in H^{m+1 / 2}(\partial \Omega), m>0$, if

$$
\|\psi\|_{H^{m+1 / 2}(\partial \Omega)}^{2}=\sum_{i=1}^{I}\left(\left\|\psi_{i}\right\|_{H^{m}(0,1)}^{2}+\left|\psi_{i}^{(m)}\right|_{1 / 2}^{2}\right)<\infty
$$

where

$$
|\phi|_{1 / 2}^{2}=\int_{0}^{1} \int_{0}^{1} \frac{\left[\phi(t)-\phi\left(t^{\prime}\right)\right]^{2}}{\left(t-t^{\prime}\right)^{2}} d t d t^{\prime}
$$

We note that $H^{m+1 / 2}(\partial \Omega)$ can be defined equivalently as the interpolation space [5]

$$
H^{m+1 / 2}(\partial \Omega)=\left[H^{m}(\partial \Omega), H^{m+1}(\partial \Omega)\right]_{1 / 2,2} \text {. }
$$

To define weighted Sobolev spaces for functions on $\partial \Omega$, let $\nu=\nu(i)$ and $\mu=\mu(i)$ be integers such that

$$
J_{i}(0)=z_{\nu}, \quad J_{i}(1)=z_{\mu}
$$

With $\beta \in R^{I}$ and $m \geqslant 0$, we define $W^{m, \beta}(\Omega)$ as the space of functions $\psi$ such that

$$
\|\psi\|_{W^{m, \beta}(\partial \Omega)}^{2}=\sum_{i=1}^{I}\left\|\psi_{i}\right\|_{m, \beta, i}^{2}<\infty
$$

where

$$
\|\phi\|_{m, \beta, i}^{2}=\sum_{j=0}^{m} \int_{0}^{1}\left[t^{\beta_{s}-m+j}(1-t)^{\beta_{\mu}-m+j}\right]^{2}\left|\phi^{(j)}(t)\right|^{2} d t
$$


Further, we let $W^{m+1 / 2, \beta}(\partial \Omega)$ denote the space with the norm

$$
\|\psi\|_{W^{m+1 / 2, \beta(\partial \Omega)}}^{2}=\|\psi\|_{W^{m, \beta-\theta / 2}(\partial \Omega)}^{2}+\sum_{i=1}^{I}\left|t^{\beta_{r}}(1-t)^{\beta_{\mu}} \psi_{i}^{(m)}\right|_{1 / 2}^{2},
$$

where $|\cdot|_{1 / 2}$ is defined by (2.3). The following interpolation result is obtained from [11].

LEMMA 2.3. If $m \geqslant 0$ and $\beta=\frac{1}{2}\left(\beta_{1}+\beta_{2}\right)$, then

$$
W^{m+1 / 2, \beta}(\partial \Omega)=\left[W^{m, \beta_{1}}(\partial \Omega), W^{m+1, \beta_{2}}(\partial \Omega)\right]_{1 / 2,2}
$$

In Section 5 we need the following trace inequality:

LEMMA 2.4. If $u \in W^{m, \beta}(\Omega), m \geqslant 1$, then

$$
\left\|u_{\mid \partial \Omega}\right\|_{W^{m-1 / 2, \beta(\partial \Omega)}} \leqslant C\|u\|_{W^{m, \beta(\Omega)}} .
$$

Proof. In [8] it is proven that if $u \in W^{1, \beta}(\Omega)$, then

$$
\left\|u_{\mid \partial \Omega}\right\|_{W^{0, \beta-\theta / 2}(\partial \Omega)}<C\|u\|_{W^{1, \beta(\Omega)}} .
$$

From this it is easily concluded that, if $u \in W^{m, \beta}(\Omega), m>1$, then

$$
\left\|u_{\mid \partial \Omega}\right\|_{W^{m-1, \beta-0 / 2}(\partial \Omega)} \leqslant C\|u\|_{W^{m, \beta}(\Omega)} \text {. }
$$

Now let $D_{m-1} u$ be any partial derivative of $u$ of order $m-1$, and let $\psi_{i}(t)=$ $\left(D_{m-1} u\right)\left(J_{i}(t)\right), i=1, \ldots, I$. Then

$$
\sum_{i=1}^{I}\left|t^{\beta_{r}}(1-t)^{\beta_{\mu}} \psi_{i}\right|_{1 / 2}^{2}<C\left\|\varphi_{\beta} D_{m-1} u_{\mid \partial \Omega}\right\|_{H^{1 / 2}(\partial \Omega)}^{2} .
$$

Using the trace properties of Sobolev spaces $H^{m}(\Omega)$ (cf. [1]) the right side can here be further estimated as

$$
\left\|\varphi_{\beta} D_{m-1} u_{\mid \partial \Omega}\right\|_{H^{1 / 2}(\partial \Omega)}<C\left\|\varphi_{\beta} D_{m-1} u\right\|_{H^{1}(\Omega)} .
$$

The asserted inequality now follows by comparing (2.5) with (2.7) through (2.9) and noting that

$$
\left\|\varphi_{\beta} D_{m-1} u\right\|_{H^{1}(\Omega)} \leqslant C\|u\|_{W^{m, \beta}(\Omega)} .
$$

We state finally a regularity result for the solution of problem (1.1). For the proof, see [8].

THEOREM 2.1. Let $m>2$ and let $\beta \in R^{I}$ be such that $\beta_{i}>0, m-1>\beta_{i}>m-$ $1-\pi / \omega_{i}, i=1, \ldots, I$. Further, let $f$ and $g$ in (1.1) be such that $f=f_{1}+f_{2}$, $f_{1} \in H^{m-2}(\Omega), f_{2} \in W^{m-2, \beta}(\Omega)$, and $g=g_{1}+g_{2}, g_{1} \in H^{m}(\Omega), g_{2} \in W^{m, \beta}(\Omega)$. Then problem (1.1) has in $H^{1}(\Omega)$ a unique weak solution $u$ which satisfies $u=u_{1}+u_{2}$, $u_{1} \in H^{m}(\Omega), u_{2} \in W^{m, \beta}(\Omega)$,

$$
\begin{aligned}
\left\|u_{1}\right\|_{H^{m}(\Omega)}+\left\|u_{2}\right\|_{W^{m, \beta}(\Omega)} & \\
& <C\left\{\left\|f_{1}\right\|_{H^{m-2}(\Omega)}+\left\|f_{2}\right\|_{W^{m-2, \beta}(\Omega)}+\left\|g_{1}\right\|_{H^{m}(\Omega)}+\left\|g_{2}\right\|_{W^{m, \beta}(\Omega)}\right\} .
\end{aligned}
$$

3. The Local Stability Conditions. In this section we state a number of conditions that are sufficient for the uniform stability of the Lagrange multiplier method. These were stated already in [10], but as they will be referred to in the sequel, we repeat then here for convenience.

Suppose we are given a partitioning $\tau^{h}(\partial \Omega)$ of $\partial \Omega$, consisting of connected smooth arcs, a finite-dimensional subspace $N^{h} \subset L_{2}(\partial \Omega)$ associated to $\tau^{h}(\partial \Omega)$ in 
the manner described below, and another finite-dimensional space $M^{\boldsymbol{h}} \subset H^{1}(\Omega)$. If $S \subset R^{n}$, we let $d(S)$ denote the diameter of $S$, and we let $K, \Lambda$, and $L$ be some fixed positive parameters, with $L$ an integer. Further, we associate to each pair $\left\{S_{i}, S_{j}\right\} \subset \tau^{h}(\partial \Omega)$ a number $n_{i j}$, defined as the minimum number of subsets $S \in$ $\tau^{h}(\partial \Omega)$ that have to be crossed when passing from a point in $S_{i}$ to a point of $S_{j}$ along $\partial \Omega$.

In the above notation, the sufficient stability conditions are as follows:

A1. To the partitioning $\tau^{h}(\partial \Omega)=\left\{S_{1}, \ldots, S_{\nu}\right\}$ there corresponds a collection $\left\{\bigodot_{1}, \ldots, \bigodot_{\nu}\right\}$ of discs $\bigodot_{j} \subset R^{2}$, such that, for each $j$, the center of $\bigodot_{j}$ is in $S_{j}$, $d\left(\mathcal{C}_{j}\right) \geqslant 8 K d\left(S_{j}\right)$, and, for each $\mathcal{C} \in\left\{\mathcal{C}_{j}\right\}, \mathcal{C} \cap \mathcal{C}_{j} \neq \varnothing$ for at most $L$ spheres $\bigodot_{j} \in\left\{\mathcal{C}_{1}, \ldots, \mathcal{C}_{\nu}\right\}$.

A2. For all pairs $\left\{S_{i}, S_{j}\right\} \subset \tau^{h}(\partial \Omega)$,

$$
\frac{d\left(S_{i}\right)}{d\left(S_{j}\right)}<K\left(1+n_{i j}\right)^{\Lambda} .
$$

A3. For each $S_{j} \in \tau^{h}(\partial \Omega)$, there exists $\phi_{j} \in N^{h}$ such that $\operatorname{supp}\left\{\phi_{j}\right\} \supseteq S_{j}$, $d\left(\operatorname{supp}\left\{\phi_{j}\right\}\right) \leqslant K d\left(S_{j}\right)$, and

$$
\left|\int_{\partial \Omega} \phi_{j} d s\right|^{2}>K^{-1} d\left(S_{j}\right) \int_{\partial \Omega} \phi_{j}^{2} d s .
$$

A4. There exists a basis $\left\{\psi_{1}, \ldots, \psi_{\mu}\right\}$ of $N^{h}$ and a set $\left\{v_{1}, \ldots, v_{\mu}\right\} \subset M^{h}$ such that

(i) For all $i, 1 \leqslant i \leqslant \mu$, if $\operatorname{supp}\left\{\psi_{i}\right\} \cap S_{j} \neq \varnothing$ and $\operatorname{supp}\left\{\psi_{i}\right\} \cap S_{k} \neq \varnothing, S_{j}, S_{k} \in$ $\tau^{h}(\partial \Omega)$, then $n_{j, k}<L$.

(ii) For all $i, 1 \leqslant i<\mu, v_{i} v_{j} \neq 0$ for at most $L$ functions $v_{j}, 1<j<\mu$.

(iii) If $S_{j} \in \tau^{h}(\partial \Omega)$, then

$$
\Lambda_{j}=\left\{i ; \psi_{i} \neq 0 \text { on } S_{j}\right\}=\left\{i ; v_{i} \not \geq 0 \text { on } S_{j}\right\}
$$

(iv) If $S_{j} \in \tau^{h}(\partial \Omega)$ and $v_{i} \neq 0$ on $S_{j}$, then

$$
K^{-1} d\left(S_{j}\right) \int_{\Omega}\left|D^{1} v_{i}\right|^{2} d x<\left\|v_{i}\right\|_{L_{2}\left(S_{j}\right)}^{2}<K\left\|\psi_{i}\right\|_{L_{2}\left(S_{j}\right)}^{2}
$$

(v) If $\psi=\Sigma_{1}^{\mu} \beta_{i} \psi_{i}, v=\Sigma_{1}^{\mu} \beta_{i} v_{i}, \beta_{i} \in R^{1}, S_{j} \in \tau^{h}(\partial \Omega)$, and $\Lambda_{j}$ is as in (iii), then

$$
\begin{aligned}
& K \sum_{i \in \Lambda_{j}} \beta_{i}^{2}\left\|\psi_{i}\right\|_{L_{2}\left(S_{j}\right)}^{2}>\|\psi\|_{L_{2}\left(S_{j}\right)}^{2}>K^{-1} \sum_{i \in \Lambda_{j}} \beta_{i}^{2}\left\|\psi_{i}\right\|_{L_{2}\left(S_{j}\right)}^{2}, \\
& K \sum_{i \in \Lambda_{j}} \beta_{i}^{2}\left\|v_{i}\right\|_{L_{2}\left(S_{j}\right)}^{2} \geqslant\|v\|_{L_{2}\left(S_{j}\right)}^{2} \geqslant K^{-1} \sum_{i \in \Lambda_{j}} \beta_{i}^{2}\left\|v_{i}\right\|_{L_{2}\left(S_{j}\right)}^{2},
\end{aligned}
$$

and

$$
\int_{S_{j}} \psi v d s \geqslant K^{-1}\|\psi\|_{L_{2}\left(S_{j}\right)}^{2}
$$

We state below a consequence of the above assumptions, which will be of basic importance in what follows. First, let us define on $\left[H^{1}(\Omega) \times L_{2}(\partial \Omega)\right]^{2}$ a bilinear form $B$ as

$$
\mathscr{B}(u, \psi ; v, \phi)=\int_{\Omega} \sum_{i=1}^{2} \frac{\partial u}{\partial x_{i}} \frac{\partial v}{\partial x_{i}} d x+\int_{\partial \Omega}(u \phi+v \psi) d s
$$


so that (1.4) can be rewritten as

$$
\mathscr{B}\left(u_{h}, \psi_{h} ; v, \phi\right)=\int_{\Omega} f v d x+\int_{\partial \Omega} g \phi d s, \quad(v, \phi) \in M^{h} \times N^{h} .
$$

Further, let us introduce on $H^{1}(\Omega) \times L_{2}(\partial \Omega)$ the weighted norm

$$
\|(u, \psi)\|_{\tau^{h}}^{2}=\int_{\Omega}\left|D^{1} u\right|^{2} d x+\sum_{j} d\left(S_{j}\right)^{-1} \int_{S_{j}} u^{2} d s+\sum_{j} d\left(S_{j}\right) \int_{S_{j}} \psi^{2} d s,
$$

where the sums are over all $S_{j} \in \tau^{h}(\partial \Omega)$. Then we have, by slightly modifying the stability result of [10],

THEOREM 3.1. Let $\tau^{h}(\partial \Omega), M^{h}$, and $N^{h}$ be such that the assumptions A1 through A4 are satisfied for some finite $K, \Lambda$, and $L$. Then, if $d(S)<h_{0}$ for all $S \in \tau^{h}(\partial \Omega)$, where $h_{0}=h_{0}(\Omega) \in(0,1)$, we have

$$
\inf _{(u, \psi) \in M^{h} \times N^{h}} \sup _{(v, \phi) \in M^{h} \times N^{h}} \frac{\Re(u, \psi ; v, \phi)}{\|(u, \psi)\|_{\tau^{h}}\|(v, \phi)\|_{\tau^{h}}}>C>0,
$$

where $C$ depends on $\gamma, K, \Lambda$, and $L$.

4. The Finite Element Subspaces. We will now introduce partitionings and subspaces, which meet with the assumptions of Section 3. First, a family of triangulations of $\Omega$ is introduced. To this end, let $\beta \in R^{I}$ be given so that $0<\beta_{i}<1, i=1, \ldots, I$, and assume that for each $h, 0<h<1$, we are given a collection $\tau_{\beta}^{h}(\Omega)$ of open, disjoint subsets of $\Omega$, such that $\cup_{T \in \tau_{\beta}^{h}(\Omega)} \bar{T}=\bar{\Omega}$ and so that each $T \in \tau_{\beta}^{h}(\Omega)$ is either a triangle or a triangle with one or two curved sides on $\partial \Omega$. We will further assume that for some positive constant $\varepsilon$, the following conditions hold for all $h \in(0,1)$.

B. (i) Each $T \in \tau_{\beta}^{h}(\Omega)$ contains a sphere of radius $\rho>\varepsilon d(T)$.

(ii) If $T \in \tau_{\beta}^{h}(\Omega)$ and $z_{i} \notin \bar{T}, i=1, \ldots, I$, then

$$
\varepsilon h \max _{x \in \bar{T}} \varphi_{\beta}(x)<d(T)<\varepsilon^{-1} h \min _{x \in \bar{T}} \varphi_{\beta}(x) .
$$

(iii) If $T \in \tau_{\beta}^{h}(\Omega)$ and $z_{i} \in \bar{T}$, then

$$
\varepsilon h \max _{x \in \bar{T}} \varphi_{\beta}(x)<d(T)<\varepsilon^{-1} h \max _{x \in \bar{T}} \varphi_{\beta}(x) .
$$

Here $\varphi_{\beta}$ refers to (2.1).

For a polygonal domain, triangulations of the above type were discussed previously in [4]. We cite from [4] that the number of triangles in a triangulation $\tau_{\beta}^{h}(\Omega)$ is bounded by $C h^{-2}$, where $C$ depends on $\Omega$ and $\varepsilon$, and $\beta$. We also point out the following implication of $\mathrm{B}(\mathrm{iii})$ :

$$
T \in \tau_{\beta}^{h}(\Omega) \& z_{i} \in \bar{T} \Rightarrow d(T)<C h^{1 /\left(1-\beta_{i}\right)} .
$$

To each $\tau_{\beta}^{h}(\Omega)$ we associate the finite element spaces $M_{\beta, k}^{h}, k=1,2, \ldots$, defined as subspaces of $H^{1}(\Omega)$ such that if $u \in M_{\beta, k}^{h}$ and $T \in \tau_{\beta}^{h}(\Omega)$, then $u_{\mid T}$ is a polynomial of degree at most $k$.

We let $\tau_{\beta}^{h}(\partial \Omega)$ denote the boundary partitioning induced by $\tau_{\beta}^{h}(\Omega)$, i.e., $\tau_{\beta}^{h}(\partial \Omega)$ consists of the sides on $\partial \Omega$ of the triangles in $\tau_{\beta}^{h}(\Omega)$. We associate to $\tau_{\beta}^{h}(\partial \Omega)$ the subspaces $N_{\beta, k}^{h} \subset L_{2}(\partial \Omega), k=1,2, \ldots$ First, assuming the notation

$$
\tau_{\beta}^{h}(\partial \Omega)=\left\{\Gamma_{i, j} \subset \partial \Omega ; \Gamma_{i j}=J_{i}\left(I_{i, j}\right), i=1, \ldots, I, j=1, \ldots, m_{i}\right\},
$$


where $J_{i}$ is as in (2.1) and

$$
I_{i, j}=\left(t_{j-1}^{i}, t_{j}^{i}\right) ; \quad 0=t_{0}^{i}<t_{1}^{i}<\cdots<t_{m_{1}}^{i}=1,
$$

let $N_{\beta, k, i}^{h}, 1<i<I$, denote the maximal subspace of $C^{0}[0,1]$ such that if $\phi \in$ $N_{\beta, k, i}^{h}$, then (i) if $k=1$, then $\phi_{\mid I_{i, j}}$ is a constant for $j=1$ and $j=m_{i}$ and a polynomial of degree unity for $2<j<m_{i}-1$; (ii) if $k>2$, then $\phi_{\mid I_{i},}$ is a polynomial of degree $k-1$ for $1 \leqslant j \leqslant m_{i}$. Then define

$$
N_{\beta, k}^{h}=\left\{\psi \in L_{2}(\partial \Omega) ; \phi(t)=\psi\left(J_{i}(t)\right) \in N_{\beta, k, i}^{h}, i=1, \ldots, I\right\} .
$$

In order to verify the assumptions Al through A4 for the above subspaces and partitionings, set $M^{h}=M_{\beta, k}^{h}, N^{h}=N_{\beta, k}^{h}$, and $\tau^{h}(\partial \Omega)=\left\{\partial T \cap \partial \Omega, T \in \tau_{\beta}^{h}(\Omega), T\right.$ has at least one side on $\partial \Omega\}$. First, we easily conclude from B(ii) that, if $S \in \tau^{h}(\partial \Omega)$

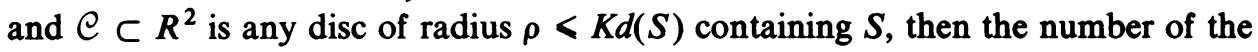
sets $S_{j} \in \tau^{h}(\partial \Omega)$ that intersect $\mathcal{C}$ is bounded by $C K$, where, if $h$ is small enough (depending on $\Omega$ ), $C$ depends only on $\beta$ and $\varepsilon$. In view of this, there exists for any given $\beta, \varepsilon, K$, a finite $L$ such that $A 1$ is satisfied for all $h \in\left(0, h_{0}\right), h_{0}=h_{0}(\Omega) \in$ $(0,1)$.

The verification of $A 2$ is also easy: using $B(i i)$ and (iii), a simple calculation shows that $A 2$ is satisfied if $K$ is large enough (depending on $\beta, \varepsilon$ ) and

$$
\Lambda=\max _{i=1, \ldots, I} \frac{\beta_{i}}{1-\beta_{i}} .
$$

To verify A3, let $S \in \tau^{h}(\partial \Omega)$ be given and let $S_{1} \in \tau^{h}(\partial \Omega)$ be a subset adjacent to $S$ on $\partial \Omega$. Then there exists a nonnegative function $\phi \in N^{h}$ such that $\operatorname{supp}\{\phi\}$ $\subset \overline{S_{1} \cup S}$. By a scaling argument, we have the inequality $\left|\int_{\partial \Omega} \phi d s\right|^{2}>$ $C d(S) \int_{\partial \Omega} \phi^{2} d s$, where $C>0$ depends on $k$ and on the constant $K$ in A2. Hence, A3 is satisfied.

We come finally to the assumption A4.

LeMmA 4.1. Let $\beta \in R^{I}$ be such that $0<\beta_{i}<1, i=1, \ldots, I$. Then there exist finite $K$ and $L$ depending on $\varepsilon$ and $\beta$ such that $M^{h}=M_{\beta, k}^{h}$ and $N^{h}=N_{\beta, k}^{h}$ satisfy A4 for all $h \in\left(0, h_{0}\right), h_{0}=h_{0}(\Omega) \in(0,1)$.

Proof. We define first a linear mapping $U: N^{h} \rightarrow M^{h}$. To this end, let $\psi \in N^{h}$ be given and let us associate to each $T \in \tau_{\beta}^{h}(\Omega)$ a polynomial $p_{T}=p_{T}(\psi)$ (on $R^{2}$ ) such that

$$
(U \psi)(x)=p_{T}(x), \quad x \in T \in \tau_{\beta}^{h}(\Omega) .
$$

We now define the polynomials $p_{T}$. First, consider a triangle $T \in \tau_{\beta}^{h}(\Omega)$ which has a side $S$ on $\partial \Omega$. We choose a cartesian coordinate system $\left\{x_{1}, x_{2}\right\}$ so that $S$ has the parametrization

$$
S=\left\{\left(x_{1}, x_{2}\right) ; x_{2}=F\left(x_{1}\right), x_{1} \in(0, d)\right\},
$$

where $F$ is a smooth function satisfying

$$
F(0)=F(d)=0, \quad\left|F\left(x_{1}\right)\right|+d\left|F^{\prime}\left(x_{1}\right)\right|<C d^{2}, \quad x_{1} \in(0, d),
$$

with $C$ independent of $S$. Now if $S$ does not touch a corner of $\partial \Omega$, we require that $p_{T}=p_{T}\left(x_{1}, x_{2}\right)$ satisfies

$$
p_{T}(i d / k, 0)=\psi(i d / k, F(i d / k)), \quad i=0, \ldots, k .
$$


In the remaining cases, i.e., one endpoint of $S$ is a corner of $\partial \Omega$, we choose the coordinates in (4.5) so that the corner is at $(0,0)$, and set

$$
p_{T}(0,0)=0, \quad p_{T}(i d / k, 0)=(i / k) \psi(i d / k, F(i d / k)), \quad i=1, \ldots, k .
$$

Consider now an arbitrary $T \in \tau_{\beta}^{h}(\Omega)$. Recall (cf. [7]) that any polynomial of degree $<k$ on $R^{2}$ is defined uniquely by its values on a set $\sigma_{T}$ given by

$$
\sigma_{T}=\left\{\frac{1}{k} \sum_{i=1}^{3} \nu_{i} a_{i} ; \nu_{i} \in\{0,1, \ldots, k\}, \sum_{i=1}^{3} \nu_{i}=k\right\},
$$

where $a_{i}$ are the vertices of $T$. Now, if $T$ has a side on $\partial \Omega$ and if $x \in \sigma_{T}$ is located on the line joining the two vertices of $T$ on $\partial \Omega$, then we define $p_{T}(x)$ as above. At the remaining points $x \in \sigma_{T}$ we set $p_{T}(x)=0$. Then each $p_{T}$ is uniquely defined. Moreover, it is easy to see that the mapping $U$ defined by (4.4) is linear and into $M^{h}$.

We establish next some inequalities for the mapping $U$. Let $\psi \in N^{h}$ be given, let $T \in \tau_{\beta}^{h}(\Omega)$ have a side $S$ on $\partial \Omega$, parametrized as in (4.5)-(4.6), and let $\phi(x)=\psi(x)$, $x \in S$, if $S$ does not touch a corner of $\Omega$, or $\phi(x)=\phi\left(x_{1}, x_{2}\right)=\left(x_{1} / d\right) \psi\left(x_{1}, x_{2}\right)$, $x \in S$, if $(0,0)$ is a corner of $\Omega$.

Let us first note that, by (4.6),

$$
\begin{aligned}
\int_{0}^{d}\left[U \psi\left(x_{1}, 0\right)\right. & \left.-U \psi\left(x_{1}, F\left(x_{1}\right)\right)\right]^{2} d x_{1} \\
= & \int_{0}^{d}\left[\int_{0}^{F\left(x_{1}\right)} \frac{\partial}{\partial x_{2}} U \psi\left(x_{1}, x_{2}\right) d x_{2}\right]^{2} d x_{1}<C d^{2} \int_{T}|\nabla U \psi|^{2} d x .
\end{aligned}
$$

Also, since $U \psi\left(x_{1}, 0\right)$ is the Lagrange interpolant of $\phi\left(x_{1}, F\left(x_{1}\right)\right)$,

$$
\begin{aligned}
& \int_{0}^{d}\left[U \psi\left(x_{1}, 0\right)\right.\left.-\phi\left(x_{1}, F\left(x_{1}\right)\right)\right]^{2} d x_{1} \\
&<C d^{2 k+2} \int_{0}^{d}\left[\left(\frac{d}{d x_{1}}\right)^{k+1} \phi\left(x_{1}, F\left(x_{1}\right)\right)\right]^{2} d x_{1}<C_{1} d^{2} \int_{S} \psi^{2} d s
\end{aligned}
$$

Here the last inequality follows from an inverse inequality for the space $N^{h}$. (Recall that $\psi$ comes from a polynomial of degree $<k-1$ if $S$ touches a corner of $\Omega$, and from a polynomial of degree $<k$ otherwise.)

Using (4.7) and (4.8) we obtain

$$
\begin{aligned}
\left|\int_{S}(U \psi-\phi) \psi d s\right|= & \left|\int_{0}^{d}[(U \psi-\phi) \psi]\left(x_{1}, F\left(x_{1}\right)\right) \frac{d s}{d x_{1}} d x_{1}\right| \\
= & \mid \int_{0}^{d}\left[U \psi\left(x_{1}, F\left(x_{1}\right)\right)-U \psi\left(x_{1}, 0\right)\right] \psi\left(x_{1}, F\left(x_{1}\right)\right) \frac{d s}{d x_{1}} d x_{1} \\
& \quad+\int_{0}^{d}\left[U \psi\left(x_{1}, 0\right)-\phi\left(x_{1}, F\left(x_{1}\right)\right)\right] \psi\left(x_{1}, F\left(x_{1}\right)\right) \frac{d s}{d x_{1}} d x_{1} \mid \\
< & C d\left\{\int_{T}|\nabla U \psi|^{2} d x\right\}^{1 / 2}\left\{\int_{S} \psi^{2} d s\right\}^{1 / 2}+C d \int_{S} \psi^{2} d s .
\end{aligned}
$$

On the other hand, by the equivalence of norms in a finite-dimensional space, we have

$$
\int_{S} \psi \phi d s>C \int_{S} \psi^{2} d s
$$


for $d$ sufficiently small. Combining the last two inequalities we obtain, for $d$ small enough,

$$
\int_{S}(U \psi) \psi d s \geqslant C \int_{S} \psi^{2} d s-C_{1} d^{2} \int_{T}|\nabla U \psi|^{2} d x
$$

Now, if $S$ is the only side of $T$ on $\partial \Omega$, then B(i) and (4.8) imply that

$$
d(T) \int_{T}|\nabla U \psi|^{2} d x \leqslant C \int_{0}^{d}\left[U \psi\left(x_{1}, 0\right)\right]^{2} d x_{1}<C_{1} \int_{S} \psi^{2} d s,
$$

where $C_{1}$ depends on $k$ and $\varepsilon$. More generally, if $T \in \tau_{\beta}^{h}(\Omega)$ is a triangle that touches $\partial \Omega$, we conclude from a similar argument that

$$
d(T) \int_{T}|\nabla U \psi|^{2} d x \leqslant C \int_{S}(U \psi)^{2} d s<C_{1} \int_{S} \psi^{2} d s,
$$

where $S=\partial T \cap \partial \Omega$ if $T$ has one or two sides on $\partial \Omega$, and otherwise $S=S_{1} \cup S_{2}$, where $S_{i} \in \tau^{h}(\partial \Omega)$ are such that $\bar{S}_{1} \cap \bar{S}_{2}$ is a vertex of $T$. Combining these inequalities, we see that

$$
\begin{aligned}
\int_{S}(U \psi) \psi d s \geqslant\left(C-C_{1} h\right) \int_{S} \psi^{2} d s> & C_{2} \int_{S} \psi^{2} d s, \\
& h \text { small enough, } S=\partial T \cap \partial \Omega \in \tau^{h}(\partial \Omega), \psi \in N^{h} .
\end{aligned}
$$

Also, since $U \psi_{\mid T}=0$ if $T$ does not touch $\partial \Omega$, we obtain

$$
\begin{aligned}
\int_{\Omega}|\nabla U \psi|^{2} d x & \leqslant C \sum_{S \in \tau^{h}(\partial \Omega)} d(S)^{-1} \int_{S}(U \psi)^{2} d S \\
& \leqslant C_{1} \sum_{S \in \tau^{h}(\partial \Omega)} d(S)^{-1} \int_{S} \psi^{2} d s .
\end{aligned}
$$

Using (4.9) and (4.10), it is now easy to complete the proof of Lemma 4.1. First, introduce a basis $\left\{\psi_{j}\right\}$ of $N^{h}$ such that A4(i) is satisfied for some finite $L$. Such a basis is easily formed from locally supported functions. We then define the set $\left\{v_{j}\right\} \subset M^{h}$ so that $v_{j}=U \psi_{j}$, where $U$ is the linear mapping constructed above. The validity of A4(ii) is then clear, and the inequalities in A4(iv) and (v) are easily proved using scaling arguments and (4.9) and (4.10).

We conclude this section by stating a convergence result for the Lagrange multiplier method (1.4) when $M^{h}=M_{\beta, k}^{h}, N^{h}=N_{\beta, k}^{h}$. We need the following result.

LEMMA 4.2. Let $\tau_{\beta}^{h}(\partial \Omega)=\left\{S_{1}, \ldots, S_{\nu}\right\}$ and let $r>-\frac{1}{2}$. Then if $h \in\left(0, h_{0}\right)$, $h_{0}=(\Omega) \in(0,1)$, and $\psi \in N_{\beta, k}^{h}$ or $\psi \in M_{\beta, k \mid \partial \Omega}^{h}, 0<\beta_{i}<1, i=1, \ldots, I$, there exist the positive constants $C_{1}$ and $C_{2}$ depending on $k, \varepsilon$ and $r \beta$ such that

$$
C_{1} h^{2 r} \int_{\partial \Omega} \varphi_{\beta}^{2 r} \psi^{2} d s<\sum_{j=1}^{\nu} d\left(S_{j}\right)^{2 r} \int_{S_{j}} \psi^{2} d s<C_{2} h^{2 r} \int_{\partial \Omega} \varphi_{\beta}^{2 r} \psi^{2} d s .
$$

Proof. If $\overline{S_{j}}$ does not contain any of the corners of $\partial \Omega$, then B(i) and B(ii) imply that, if $h$ is small enough and $\psi \in L_{2}(\partial \Omega)$, then

$$
C_{1} h^{2 r} \int_{S_{j}} \varphi_{\beta}^{2 r} \psi^{2} d s \leqslant d\left(S_{j}\right)^{2 r} \int_{S_{j}} \psi^{2} d s \leqslant C_{2} h^{2 r} \int_{S_{j}} \varphi_{\beta}^{2 r} \psi^{2} d s
$$


where $C_{1}$ and $C_{2}$ depend on $\varepsilon$. Assume then that $z_{i} \in \overline{S_{j}}, 1<i<I$. Using the fact that

$$
C_{1} \int_{0}^{1} t^{\alpha} p^{2} d t<\int_{0}^{1} p^{2} d t<C_{2} \int_{0}^{1} t^{\alpha} p^{2} d t
$$

for $\alpha>-1$ and for any polynomial $p$ of degree $k$, with $C_{1}$ and $C_{2}$ depending on $\alpha$ and $k$, we conclude by scaling that if $\psi \in N_{\beta, k}^{h}$ or $\psi \in M_{\beta, k \mid \partial \Omega}^{h}$ and $h$ is sufficiently small, then

$$
\begin{aligned}
C_{1} d\left(S_{j}\right)^{-\alpha} \int_{S_{j}} \mid x & -\left.z_{i}\right|^{\alpha} \psi^{2} d s \\
& <\int_{S_{j}} \psi^{2} d s<C_{2} d\left(S_{j}\right)^{-\alpha} \int_{S_{j}}\left|x-z_{i}\right|^{\alpha} \psi^{2} d s, \quad \alpha>-1 .
\end{aligned}
$$

Choosing $\alpha=2 r \beta_{i}$ and noting that, by B(i) and (4.1),

$$
C_{1} h^{2 r}<d\left(S_{j}\right)^{2 r\left(1-\beta_{i}\right)}<C_{2} h^{2 r}
$$

we again obtain (4.11), with $C_{1}$ and $C_{2}$ now depending on $\varepsilon, r \beta_{i}$ and $k$.

Since (4.11) holds for all $S_{j} \in \tau_{\beta}^{h}(\partial \Omega)$, the assertion follows by summing over j.

We note that, by Lemma 2.4 and Lemma 2.1 , we have the inequality

$$
\int_{\partial \Omega} \varphi_{\beta}^{-1} u^{2} d s<C\|u\|_{H^{1}(\Omega)}^{2}, \quad u \in H^{1}(\Omega) .
$$

In view of this, if we define

$$
\|(u, \psi)\|_{\beta, h}^{2}=\int_{\Omega}\left|D^{1} u\right|^{2} d x+h^{-1} \int_{\partial \Omega} \varphi_{\beta}^{-1} u^{2} d s+h \int_{\partial \Omega} \varphi_{\beta} \psi^{2} d s,
$$

then $\|\cdot\|_{\beta, h}$ is a norm in the space $H^{1}(\Omega) \times W^{0, \beta / 2}(\partial \Omega)$. We denote the normed space $\left(H^{1}(\Omega) \times W^{0, \beta / 2}(\partial \Omega),\|\cdot\|_{\beta, h}\right)$ by $X_{\beta, h}$.

Let the bilinear form $\mathscr{B}$ be as in (3.1). We replace from here on the weak formulation (1.2) by the following more convenient variational problem:

$$
(u, \psi) \in X_{\beta, h}: \mathscr{B}(u, \psi ; v, \phi)=\int_{\Omega} f v d x+\int_{\partial \Omega} g \phi d s, \quad(v, \phi) \in X_{\beta, h} .
$$

In the sequel it is of importance that (4.12) is solvable under weaker assumptions on $f$ and $g$ than (1.2). From (3.1) one concludes immediately that the bilinear form $\mathscr{B}$ is bounded on $X_{\beta, h} \times X_{\beta, h}$ :

$$
|\Re(u, \psi ; v, \phi)| \leqslant\|(u, \psi)\|_{\beta, h}\|(v, \psi)\|_{\beta, h} .
$$

Moreover, by Theorem 3.1 and Lemma 4.2,

$$
\inf _{(u, \psi) \in M_{\beta, k}^{h} \times N_{\beta, k}^{h}} \sup _{(v, \phi) \in M_{\beta, k}^{h} \times N_{\beta, k}^{h}} \frac{\Re(u, \psi ; v, \phi)}{\|(u, \psi)\|_{\beta, h}\|(v, \phi)\|_{\beta, h}}>C>0 .
$$

From (1.4) and (4.12) through (4.14) we conclude, by classical arguments (see [3]), the following result:

TheOREM 4.1. Assume that problem (4.11) has a solution $(u, \psi) \in H^{1}(\Omega) \times$ $W^{0, \beta / 2}(\partial \Omega), 0<\beta_{i}<1, i=1, \ldots, I$. Then, if $\left(u_{h}, \psi_{h}\right)$ is the solution of (1.4) with $M^{h}=M_{\beta, k}^{h}, N^{h}=N_{\beta, k}^{h}, h \in\left(0, h_{0}\right), h_{0}=h_{0}(\Omega) \in(0,1)$, we have the error estimate

$$
\left\|(u, \psi)-\left(u_{h}, \psi_{h}\right)\right\|_{\beta, h}<C \min _{(v, \phi) M_{\beta, k}^{h} \times N_{\beta, k}^{h}}\|(u, \psi)-(v, \phi)\|_{\beta, h},
$$

where $C$ depends on $\beta, k$ and $\varepsilon$. 
5. Rate of Convergence. We state first some lemmas which pertain to the approximation properties of polynomials in weighted Sobolev spaces. In what follows, $T$ denotes a triangle with vertices at $(0,0),(1,0)$ and $(0,1)$.

Our first lemma is from [4].

Lemma 5.1. Let $\alpha \neq 0$ and let $u$ be defined on $T$ such that $\int_{T}|x|^{\alpha}\left|D^{1} u\right|^{2} d x<\infty$. Then there exists a constant $q$, depending on $u$ and $\alpha$, and $a$ constant $C>0$, depending on $\alpha$, such that

$$
\int_{T}|x|^{\alpha-2}|u-q|^{2} d x<C \int_{T}|x|^{\alpha}\left|D^{1} u\right|^{2} d x .
$$

LeMma 5.2. Let $k$ be an integer, $k>1, \alpha \in R^{1}, \alpha \notin\{0,2, \ldots, 2 k-2\}$, and let $u$ be defined on $T$ such that $\int_{T}|x|^{\alpha}\left|D^{k} u\right|^{2} d x<\infty$. Then there exists a polynomial $p$ of degree $k-1$ and a constant $C$, depending on $\alpha, k$, such that

$$
\sum_{j=0}^{k-1} \int_{T}|x|^{\alpha+2 j-2 k}\left|D^{j}(u-p)\right|^{2} d x \leqslant C \int_{T}|x|^{\alpha}\left|D^{k} u\right|^{2} d x .
$$

Proof. We first apply Lemma 5.1 to find constants $q_{i j}, i+j=k-1$, such that

$$
\int_{T}|x|^{\alpha-2}\left(\frac{\partial^{k-1} u}{\partial x_{1}^{i} \partial x_{2}^{j}}-q_{i j}\right)^{2} d x<C \int_{T}|x|^{\alpha}\left|D^{k} u\right|^{2} d x .
$$

Then define

$$
p_{k-1}(x)=\sum_{i+j=k-1} \frac{q_{i j}}{i ! j !} x_{1}^{i} x_{2}^{j}
$$

Let $u_{k-1}=u-p_{k-1}$, and apply again Lemma 5.1 to find constants $q_{i j}, i+j=$ $k-2$, such that

$$
\int_{T}|x|^{\alpha-4}\left(\frac{\partial^{k-2} u_{k-1}}{\partial x_{1}^{i} \partial x_{2}^{j}}-q_{i j}\right)^{2} d x \leqslant C \int_{T}|x|^{\alpha-2}\left|D^{k-1} u_{k-1}\right|^{2} d x .
$$

Further, define $u_{k-2}=u_{k-1}-p_{k-2}$, where

$$
p_{k-2}=\sum_{i+j=k-2} \frac{q_{i j}}{i ! j !} x_{1}^{i} x_{2}^{j} .
$$

Continuing in this way, one finally finds the homogeneous polynomials $\boldsymbol{p}_{\boldsymbol{j}}$ of degree $j, 0<j<k-1$, such that if $u_{j}=u-\sum_{l-j}^{k-1} p_{l}$ for $0<j<k-1$, and $u_{k}=u$, then

$$
\begin{aligned}
\int_{T}|x|^{\alpha+2 j-2 k} & \left|D^{j} u_{j}\right|^{2} d x \\
& \leqslant C \int_{T}|x|^{\alpha+2 j+2-2 k}\left|D^{j+1} u_{j+1}\right|^{2} d x, \quad j=0, \ldots, k-1 .
\end{aligned}
$$

Noting that $D^{j} u_{j}=D^{j} u_{0}$, the assertion follows by taking $p=\sum_{j=0}^{k-1} p_{j}$.

Let $\Sigma_{k}$ denote the set of nodal points on $\bar{T}$ associated to a reference Lagrange element of degree $k, k \geqslant 1$ (cf. [7]). For a continuous function $u$, defined on $T$, we let $(u)_{k}$ denote the $k$ th order Lagrange interpolant to $u$, i.e., $(u)_{k}$ is a polynomial of degree $k$ such that $(u)_{k}(x)=u(x)$ for $x \in \Sigma_{k}$. We have

LEMMA 5.3. Let $k>2$, let $2 k-4<\alpha<2 k-2$, and let $u$ be defined on $T$ such that $\int_{T}|x|^{\alpha}\left|D^{k} u\right|^{2} d x<\infty$. Then (5.2) holds for a polynomial $p=(u)_{k}$. 
Proof. Let $p$ be the polynomial of Lemma 5.2. Then we have

$$
(u)_{k}=(u-p)_{k}+p .
$$

From Lemma 2.2 and from (5.2), we have

$$
\begin{aligned}
|(u-p)(x)|^{2} & \leqslant C \int_{T}|x|^{\alpha}\left|D^{k} u\right|^{2} d x, \quad x \in T, \\
(u-p)(0) & =0 .
\end{aligned}
$$

Then also $(u-p)_{k}(0)=0$, so we have

$$
\sum_{l=0}^{k-1} \int_{T}|x|^{\alpha+2 l-2 k}\left|D^{l}(u-p)_{k}\right|^{2} d x<\infty, \quad \alpha>2 k-4 .
$$

Further, by the definition of the interpolant and by (5.4),

$$
\begin{aligned}
& \sum_{l=0}^{k-1} \int_{T}|x|^{\alpha+2 l-2 k}\left|D^{l}(u-p)_{k}\right|^{2} d x \\
&<C \sum_{x \in \Sigma_{k}}|(u-p)(x)|^{2}<C_{1} \int_{T}|x|^{\alpha}\left|D^{k} u\right|^{2} d x .
\end{aligned}
$$

The assertion now follows by combining (5.3), (5.5), and (5.2) and using the triangle inequality.

Using the above results we now prove the first approximation theorem.

Theorem 5.1. Let $k \geqslant 1$, and let $\beta \in R^{I}$ be such that $0<\beta_{i}<1, i=1, \ldots, I$. Further, let $u$ be defined on $\Omega$ such that $\int_{\Omega} \varphi_{\beta}^{2 k}\left|D^{k+1} u\right|^{2} d x<\infty$. Then, if $h \in\left(0, h_{0}\right)$, $h_{0}=h_{0}(\Omega) \in(0,1)$, there exists a constant $C$, depending on $k, \beta$, such that

$$
\begin{gathered}
\min _{u \in M_{\beta, k}^{h}}\left\{\int_{T}\left|D^{1}(u-v)\right|^{2} d x+h^{-1} \int_{\partial \Omega} \varphi_{\beta}^{-1}|u-v|^{2} d s\right\} \\
<C h^{2 k} \int_{\Omega} \varphi_{\beta}^{2 k}\left|D^{k+1} u\right|^{2} d x .
\end{gathered}
$$

Proof. We begin by introducing an interpolant of $u$. Let $T$ be the reference triangle as above. Then, if $T_{j} \in \tau_{\beta}^{h}(\Omega)$, we can write

$$
T=\left(G_{j} \circ A_{j}\right)\left(T_{j}\right), \quad T_{j} \in \tau_{\beta}^{h}(\Omega),
$$

where $A_{j}$ is an affine transformation which maps the corners of $T_{j}$ onto those of $T$, and $G_{j}$ is a smooth transformation which straightens the curved sides (if any) of $A_{j}\left(T_{j}\right)$. If $\bar{T}_{j}$ contains a corner $z_{i}$ of $\partial \Omega$, then we assume $A_{j}$ to be chosen so that $A_{j}\left(z_{i}\right)=\{0\}$.

With the nodal set $\Sigma_{k}$ defined as above, let $\Sigma_{k}^{j}=\left(G_{j} \circ A_{j}\right)^{-1}\left(\Sigma_{k}\right)$, and define $u^{j}$ as the polynomial of degree $k$ on $T_{j}$ such that $u^{j}(x)=u(x)$ for $x \in \Sigma_{k}^{j}$. Since the sides of the triangles $T_{j} \in \tau_{\beta}^{h}(\Omega)$ become straight in the limit $h \rightarrow 0$, we see that, if $h$ is sufficiently small, then $u^{j}$ is uniquely defined for all $T_{j} \in \tau_{\beta}^{h}(\Omega)$. We let $u_{\beta, k}^{h} \in M_{\beta, k}^{h}$ denote the interpolant defined as

$$
u_{\beta, k}^{h}(x)=u^{j}(x), \quad x \in T_{j} \in \tau_{\beta}^{h}(\Omega) .
$$

We will first prove that, under the assumptions made,

$$
\int_{\Omega}\left|D^{1}\left(u-u_{\beta, k}^{h}\right)\right|^{2} d x \leqslant C h^{2 k} \int_{\Omega} \varphi_{\beta}^{2 k}\left|D^{k+1} u\right|^{2} d x .
$$


Let $T_{j} \in \tau_{\beta}^{h}(\Omega)$ be a triangle which does not touch any of the corners of $\partial \Omega$. Then we have the classical result

$$
\int_{T_{j}}\left|D^{1}\left(u-u^{j}\right)\right|^{2} d x \leqslant C d\left(T_{j}\right)^{2 k} \int_{T_{j}}\left|D^{k+1} u\right|^{2} d x
$$

where $C$ depends only on $k$ and $\varepsilon$ for $h$ sufficiently small. Using B(ii), this can be further written as

$$
\int_{T_{j}}\left|D^{1}\left(u-u^{j}\right)\right|^{2} d x \leqslant C h^{2 k} \int_{T_{j}} \varphi_{\beta}^{2 k}\left|D^{k+1} u\right|^{2} d x
$$

We show next that (5.8) also holds when $\bar{T}_{j}$ contains a corner $z_{i}$ of $\partial \Omega$. We note first that Lemma 5.1 holds also when $T$ is replaced by $A_{j}\left(T_{j}\right), A_{j}$ as in (5.6), provided that $h$ is small enough. Moreover, the constant $C$ in (5.1) does not depend on $j$. This follows, since $A_{j}\left(T_{j}\right)=G_{j}^{-1}(T)$, and the Jacobian of $G_{j}^{-1}$ tends to unity uniformly in $j$ as $h \rightarrow 0$. Then Lemma 5.2 holds also when $T$ is replaced by $A_{j}\left(T_{j}\right)$, and so does Lemma 5.3, if $\Sigma_{k}$ is replaced by $G_{j}^{-1}\left(\Sigma_{k}\right)$ in the definition of the interpolant $(u)_{k}$.

Now choose any $\alpha \in R^{1}$ so that $2 k-2<\alpha<2 k, \alpha>2 k \beta_{i}$. Then, using the above modifications in Lemma 5.3, we conclude that, if $\tilde{u}(x)=u\left(A_{j}^{-1}(x)\right), \tilde{u}^{j}(x)=$ $u^{j}\left(A_{j}^{-1}(x)\right), x \in A_{j}\left(T_{j}\right)$, then

$$
\int_{A_{j}\left(T_{j}\right)}|x|^{\alpha-2 k}\left|D^{1}\left(\tilde{u}-\tilde{u}^{j}\right)\right|^{2} d x \leqslant C \int_{A_{j}\left(T_{j}\right)}|x|^{\alpha}\left|D^{k+1} \tilde{u}\right|^{2} d x .
$$

This inequality is invariant in scaling. Therefore, and taking into account the assumption B(i), the affine transformation $A_{j}^{-1}$ can only introduce a dependence on $\varepsilon$ in the constant $C$. Thus, we have

$$
\int_{T_{j}}\left|x-z_{i}\right|^{\alpha-2 k}\left|D^{1}\left(u-u^{j}\right)\right|^{2} d x<C \int_{T_{j}}\left|x-z_{i}\right|^{\alpha}\left|D^{k+1} u\right|^{2} d x,
$$

where $C$ depends on $k, \alpha, \varepsilon$. Using (4.1), we have here the further estimates

$$
\begin{aligned}
\left|x-z_{i}\right|^{\alpha-2 k} & >C h^{(\alpha-2 k) /\left(1-\beta_{i}\right)}, \\
\left|x-z_{i}\right|^{\alpha} & \leqslant C\left[\varphi_{\beta}(x)\right]^{2 k} h^{\left(\alpha-2 k \beta_{i}\right) /\left(1-\beta_{i}\right)}, \quad x \in T_{j}, C>0 .
\end{aligned}
$$

Together with (5.9), these prove (5.8).

Having verified that (5.8) holds for all $T_{j} \in \tau_{\beta}^{h}(\Omega)$, it suffices to sum over $j$ to prove (5.7).

We prove next the estimate

$$
h^{-1} \int_{\partial \Omega} \varphi_{\beta}^{-1}\left|u-u_{\beta, k}^{h}\right|^{2} d s \leqslant C h^{2 k} \int_{\partial \Omega} \varphi_{\beta}^{2 k}\left|D^{k+1} u\right|^{2} d x
$$

Let $T_{j} \in \tau_{\beta}^{h}(\Omega)$ be a triangle such that $T_{j}$ has a curved side $S_{j}$ on $\partial \Omega$, and let $\tilde{u}$ and $\tilde{u}^{j}$ be defined on $A_{j}\left(T_{j}\right)=G_{j}^{-1}(T)$ as above. Assume first that $S_{j}$ does not touch any of the corners of $\partial \Omega$. Then we start from the inequality

$$
\int_{A_{j}\left(S_{j}\right)}\left|\tilde{u}-\tilde{u}^{j}\right|^{2} d s \leqslant C\left\|\tilde{u}-\tilde{u}^{j}\right\|_{H^{1}\left(A_{j}\left(T_{j}\right)\right)}^{2} \leqslant C_{1} \int_{A_{j}\left(T_{j}\right)}\left|D^{k+1} \tilde{u}\right|^{2} d x,
$$


where $C_{1}$ depends only on $k$ and $\varepsilon$ for $h$ sufficiently small. Applying to this the affine transformation $A_{j}^{-1}$, one gets

$$
d\left(T_{j}\right)^{-1} \int_{S_{j}}\left|u-u^{j}\right|^{2} d s<C d\left(T_{j}\right)^{2 k} \int_{T_{j}}\left|D^{k+1} u\right|^{2} d x,
$$

and further, using B(ii),

$$
h^{-1} \int_{S_{j}} \varphi_{\beta}^{-1}\left|u-u^{j}\right|^{2} d s \leqslant C h^{2 k} \int_{T_{j}} \varphi_{\beta}^{2 k}\left|D^{k+1} u\right|^{2} d x
$$

If $z_{i} \in \bar{S}_{j}$, we take $\alpha \in(2 k-2,2 k), \alpha>2 k \beta_{i}$, and derive first from (2.6) the inequality

$$
\begin{aligned}
& \int_{A_{j}\left(S_{j}\right)}|x|^{\alpha-2 k-1}\left|\tilde{u}-\tilde{u}^{j}\right|^{2} d s \\
& \quad<C \int_{A_{j}\left(T_{j}\right)}\left\{|x|^{\alpha-2 k-2}\left|\tilde{u}-\tilde{u}^{j}\right|^{2}+|x|^{\alpha-2 k}\left|D^{1}\left(\tilde{u}-\tilde{u}^{j}\right)\right|^{2}\right\} d x .
\end{aligned}
$$

Applying on the right side the same reasoning as above and performing the affine transformation $A_{j}^{-1}$, we conclude that

$$
\int_{S_{j}}\left|x-z_{i}\right|^{\alpha-2 k-1}\left|u-u^{j}\right|^{2} d s<C \int_{T_{j}}\left|x-z_{i}\right|^{\alpha}\left|D^{k+1} u\right|^{2} d x .
$$

Since, by (4.1),

$$
\begin{aligned}
\left|x-z_{i}\right|^{\alpha-2 k-1}>C h^{\left(\alpha-2 k-1+\beta_{i}\right) /\left(1-\beta_{i}\right)}\left[\varphi_{\beta}(x)\right]^{-1}, & \\
\left|x-z_{i}\right|^{\alpha}<C h^{\left(\alpha-2 k \beta_{i}\right) /\left(1-\beta_{i}\right)}\left[\varphi_{\beta}(x)\right]^{2 k}, & 2 k \beta_{i}<\alpha<2 k, x \in S_{j}, C>0,
\end{aligned}
$$

we again obtain the estimate (5.11).

Upon summing over $j$ in (5.11), the estimate (5.10) follows. Finally, combining (5.7) and (5.10), the proof of Theorem 5.1 is complete.

The second approximation theorem is as follows.

Theorem 5.2. Let $k \geqslant 1$, and let $\beta \in R^{I}$ be such that $0<\beta_{i}<1, i=1, \ldots, I$. Then, if $u \in W^{k+1, k \beta}(\Omega)$ and $\psi$ is defined by (1.3), there exists a constant $C$, depending on $k, \beta, \varepsilon$ and $\Omega$, such that

$$
\min _{\phi \in N_{\beta, k}^{h}}\left\{h \int_{\partial \Omega} \varphi_{\beta}|\psi-\psi|^{2} d s\right\}<C h^{2 k}\|u\|_{W^{k+1, k \beta}(\Omega)}^{2} .
$$

Proof. Consider first a function $\psi$ defined on $\partial \Omega$ and sufficiently smooth on each $\Gamma_{i}, i=1, \ldots, I$. We use the notation of (4.2) and (4.3) to define an interpolant of $\psi$ in $N_{\beta, k}^{h}$. First, let $\psi_{i}(t)=\psi\left(J_{i}(t)\right), t \in(0,1), i=1, \ldots, I$, and let $\psi_{i, k}$ denote the interpolant of $\psi_{i}$ in the partitioning $\left\{I_{j}^{(i)}\right\}_{j-1}^{m_{i}}$, defined so that (i) if $2<j<m_{i}-1$, then $\psi_{i, k \mid I_{j}^{i}}$ equals the Lagrange interpolant of $\psi_{i}$ of degree $\max \{1, k-1\}$ on $I_{j}^{i}$, and (ii) if $j=1$ or $j=m_{i}$, then $\psi_{i, k \mid I_{j}}$ is defined as a polynomial of degree $k-1$ such that

$$
\begin{aligned}
\left(\psi_{i}^{(l)}-\psi_{i, k}^{(l)}\right)\left(t_{1}^{i}-0\right) & =0, \\
\left(\psi_{i}^{(l)}-\psi_{i, k}^{(l)}\right)\left(t_{m_{i}-1}^{i}+0\right) & =0, \quad l=0, \ldots, k-1 .
\end{aligned}
$$


We let $\psi_{\beta, k}^{h}$ be a function defined on $\partial \Omega$ such that

$$
\psi_{\beta, k}^{h}(x)=\psi_{i, k}\left(J_{i}^{-1}(x)\right), \quad x \in \Gamma_{i}, i=1, \ldots, I .
$$

It is clear from the definition of $N_{\beta, k}^{h}$ that $\psi_{\beta, k}^{h} \in N_{\beta, k}^{h}$. We will establish first the interpolation error estimates

$$
\begin{aligned}
h \int_{\partial \Omega} \varphi_{\beta}\left|\psi-\psi_{\beta, k}^{h}\right|^{2} d s<C h^{2 k+1}\|\psi\|_{W^{k,(k+1 / 2) \beta}(\partial \Omega)}^{2} & \\
\psi & \in W^{k,(k+1 / 2) \beta}(\partial \Omega), k=1,2, \ldots
\end{aligned}
$$

To prove (5.13), let $\nu=\nu(i)$ and $\mu=\mu(i)$ be as in (2.4). Then B(ii) implies the inequalities

$$
C_{1} t^{\beta_{r}}(1-t)^{\beta_{\mu}}<d\left(I_{j}^{i}\right)<C_{2} t^{\beta_{r}}(1-t)^{\beta_{\mu}}, \quad t \in I_{j}^{i}, j=2, \ldots, m_{i}-1,
$$

where $C_{1}$ and $C_{2}$ depend only on $\varepsilon$. Combining these with the classical estimates of the error of Lagrange interpolation we get

$$
\begin{aligned}
& h \int_{I_{j}^{i}} t^{\beta_{r}}(1-t)^{\beta_{\mu}}\left|\psi_{i}-\psi_{i, k}\right|^{2} d t \\
& \quad<C h^{2 k+1} \int_{l_{j}^{i}} t^{(2 k+1) \beta_{r}}(1-t)^{(2 k+1) \beta_{\mu}}\left|\psi_{i}^{(k)}\right|^{2} d t, \quad j=2, \ldots, m_{i}-1 .
\end{aligned}
$$

Let us now show that (5.14) also holds for $j=1$ and $j=m_{i}$. We need the following consequence of Hardy's inequality [8]: Let $\alpha>0, k>0$, and let $f$ be defined on $(0,1)$ so that $\int_{0}^{1} t^{\alpha+2 k}\left|f^{(k)}\right|^{2} d t<\infty$, and so that $f^{(l)}(1)=0, l=0, \ldots, k-1$. Then

$$
\int_{0}^{1} t^{\alpha} f^{2} d t<C \int_{0}^{1} t^{\alpha+2 k}\left|f^{(k)}\right|^{2} d t
$$

where $C$ depends only on $k$.

Since (5.15) is invariant in scaling, we conclude from (5.12) and (5.15) that

$$
\begin{aligned}
\int_{I_{j}^{j}} t^{\beta_{2}}(1-t)^{\beta_{\mu}}\left|\psi_{i}-\psi_{i, k}\right|^{2} d t & \\
& <C \int_{I_{j}^{j}} t^{\beta_{s}+2 k}(1-t)^{\beta_{\mu}+2 k}\left|\psi_{i}^{(k)}\right|^{2} d t, \quad j \in\left\{1, m_{i}\right\} .
\end{aligned}
$$

On the other hand, B(iii) implies that

$$
t<C h^{1 /\left(1-\beta_{*}\right)}, \quad t \in I_{1}^{i},
$$

and

$$
1-t<C h^{1 /\left(1-\beta_{\mu}\right)}, \quad t \in I_{m_{i}}^{i} \text {. }
$$

Using these estimates in (5.16), it follows that (5.14) holds also for $j=1$ and $j=m_{i}$. It then suffices to sum over $i$ and $j$ to prove (5.13).

In view of (5.13), the following estimates hold:

$$
\begin{array}{rr}
\min _{\phi \in N_{\beta, k}^{h}}\left\{h \int_{\partial \Omega} \varphi_{\beta}|\psi-\phi|^{2} d s\right\} & \\
<C h^{2 k+1}\|\psi\|_{W^{k,(k+1 / 2) \beta}(\partial \Omega)}^{2}, & \psi \in W^{k,(k+1 / 2) \beta}(\partial \Omega) \\
<C h^{2 k-1}\|\psi\|_{W^{k-1,(k-1 / 2) \beta}(\partial \Omega),}^{2} & \psi \in W^{k-1,(k-1 / 2) \beta}(\partial \Omega), \\
& k=1,2, \ldots
\end{array}
$$


Here the second estimate is trivial if $k=1$, and for $k>1$ it follows from (5.13) and from the inclusion $N_{\beta, k-1}^{h} \subset N_{\beta, k}^{h}$. Upon interpolating between these estimates and using Lemma 2.3, we have

$$
\begin{aligned}
\min _{\phi \in N_{\beta, k}^{h}}\left\{h \int_{\partial \Omega} \varphi_{\beta}|\psi-\phi|^{2} d s\right\} & \\
& \leqslant C h^{2 k}\|\psi\|_{W^{k-1 / 2, k \beta}(\partial \Omega)}^{2}, \quad \psi \in W^{k-1 / 2, k \beta}(\partial \Omega), k=1,2, \ldots
\end{aligned}
$$

To complete the proof, take $\psi$ in (5.17) to be defined by (1.3), with $u \in$ $W^{k+1, k \beta}(\Omega)$. Since $n_{i}$ in (1.3) are smooth functions on each $\operatorname{arc} \Gamma_{i}, i=1, \ldots, I$, and since the operator of multiplication by a smooth function is bounded in the weighted Sobolev spaces considered, we conclude from Lemma 2.4 that $\psi$ satisfies

$$
\|\psi\|_{W^{k-1 / 2, k \beta}(\partial \Omega)} \leqslant C\|u\|_{W^{k+1, k \beta(\Omega)}} .
$$

Upon combining this with (5.17), the proof is complete.

Using the above approximation results, we can now estimate the error of the Lagrange multiplier method in terms of weighted norms.

THEOREM 5.3. Let $\beta \in R^{I}$ be such that $\beta_{i}>0,1>\beta_{i}>1-\pi / \omega_{i} k, i=$ $1, \ldots, I$. Further, let $u$ be the solution of problem (1.1) with $f=f_{1}+f_{2}, f_{1} \in$ $H^{k-1}(\Omega), f_{2} \in W^{k-1, k \beta}(\Omega)$ and $g=g_{1}+g_{2}, g_{1} \in H^{k+1}(\Omega), g_{2} \in W^{k+1, k \beta}(\Omega)$, and let $\psi$ be defined by (1.3). Then, if $h \in\left(0, h_{0}\right), h_{0}=h_{0}(\Omega) \in(0,1)$, and $M^{h}=M_{\beta, k}^{h}$, $N^{h}=N_{\beta, k}^{h}$, (1.4) has a unique solution $\left(u_{h}, \psi_{h}\right)$, and there exists a constant $C$, depending on $k, \beta, \varepsilon$ and $\Omega$, such that

$$
\begin{aligned}
\int_{\Omega} \varphi_{\beta}^{-2} \mid u & -\left.u_{h}\right|^{2} d x+h^{2} \int\left|D^{1}\left(u-u_{h}\right)\right|^{2} d x \\
& +h \int_{\partial \Omega} \varphi_{\beta}^{-1}\left|u-u_{h}\right|^{2} d s+h^{3} \int_{\partial \Omega} \varphi_{\beta}\left|\psi-\psi_{h}\right|^{2} d s \\
\leqslant & C h^{2 k+2}\left\{\left\|f_{1}\right\|_{H^{k-1}(\Omega)}^{2}+\left\|f_{2}\right\|_{W^{k-1, k \beta}(\Omega)}^{2}+\left\|g_{1}\right\|_{H^{k+1}(\Omega)}^{2}+\left\|g_{2}\right\|_{W^{k+1, k \beta}(\Omega)}^{2}\right\}^{2}
\end{aligned}
$$

Proof. Given $k$ and $\beta$, which satisfy the assumptions of the theorem, we have, by Theorem 2.1,

$$
\begin{gathered}
u=u_{1}+u_{2}, \quad u_{1} \in H^{k+1}(\Omega), u_{2} \in W^{k+1, k \beta}(\Omega) \\
\left\|u_{1}\right\|_{H^{k+1}(\Omega)}^{2}+\left\|u_{2}\right\|_{W^{k+1, k \beta}(\Omega)}^{2} \\
\leqslant C\left\{\left\|f_{1}\right\|_{H^{k-1}(\Omega)}^{2}+\left\|f_{2}\right\|_{W^{k-1, k \beta}(\Omega)}^{2}+\left\|g_{1}\right\|_{H^{k+1}(\Omega)}^{2}+\left\|g_{2}\right\|_{W^{k+1, k \beta}(\Omega)}^{2}\right\} .
\end{gathered}
$$

Also, by (1.3) and Lemma 2.4 and by the trace properties of functions in Sobolev spaces $H^{m}(\Omega), \psi$ satisfies

$$
\begin{gathered}
\psi=\psi_{1}+\psi_{2}, \quad \psi_{1} \in H^{k-1 / 2}(\partial \Omega), \psi_{2} \in W^{k-1 / 2, k \beta}(\partial \Omega), \\
\left\|\psi_{1}\right\|_{H^{k-1 / 2}(\partial \Omega)}^{2}+\left\|\psi_{2}\right\|_{W^{k-1 / 2, k \beta}(\partial \Omega)}^{2} \leqslant C\left\{\left\|u_{1}\right\|_{H^{k+1}(\Omega)}^{2}+\left\|u_{2}\right\|_{W^{k+1, k \beta}(\Omega)}^{2}\right\} .
\end{gathered}
$$

From (5.19) and (2.5), we conclude that $\psi_{2} \in W^{0, k \beta-(k-1 / 2) \theta}(\partial \Omega)$. Since $\psi_{1} \in$ $L_{2}(\partial \Omega)$ and $0 \leqslant \beta_{i}<1, i=1, \ldots, I$, it follows that $\psi \in W^{0, \beta / 2}(\partial \Omega)$. Hence, $(u, \psi)$ is the solution of problem (4.11), and the error bound of Theorem 4.1 applies to the Lagrange multiplier method. 
By Theorems 5.1 and 5.2, we have

$$
\begin{array}{r}
\min _{v \in M_{\beta, k}^{h}}\left\{\int_{\Omega}\left|D^{1}(u-v)\right|^{2} d x+h^{-1} \int_{\partial \Omega} \varphi_{\beta}|u-v|^{2} d s\right\} \\
\leqslant C h^{2 k} \int_{\Omega} \varphi_{\beta}^{2 k}\left|D^{k+1} u\right|^{2} d x
\end{array}
$$

and

$$
\min _{\phi \in N_{\beta, k}^{h}}\left\{h \int_{\partial \Omega} \varphi_{\beta}\left|\psi_{2}-\phi\right|^{2} d s\right\}<C h^{2 k}\left\|u_{2}\right\|_{W^{k+1, k \beta}(\Omega)}^{2}
$$

Also, using the same type of reasoning as in the proof of Theorem 5.2, one gets

$$
\begin{aligned}
& \min _{\phi \in N_{\beta, k}^{h}}\left\{h \int_{\partial \Omega} \varphi_{\beta}\left|\psi_{1}-\phi\right|^{2} d s\right\} \\
& \quad<C \min _{\phi \in N_{\beta, k}^{h}}\left\{h \int_{\partial \Omega}\left|\psi_{1}-\phi\right|^{2} d s\right\}<C_{1} h^{2 k}\left\|\psi_{1}\right\|_{H^{k-1 / 2}(\partial \Omega)}^{2}
\end{aligned}
$$

We finally estimate $\int_{\Omega} \varphi_{\beta}^{-2}\left|u-u_{h}\right|^{2} d x$ using a duality argument. Consider the auxiliary problem

$$
\begin{aligned}
-\Delta V & =\varphi_{\beta}^{-2}\left(u-u_{h}\right), \quad x \in \Omega, \\
V & =0, \quad x \in \partial \Omega,
\end{aligned}
$$

the solution of which satisfies [8]

$$
V \in W^{2, \beta}(\Omega)
$$

$$
\|V\|_{W^{2, \beta(\Omega)}} \leqslant C\left\|\varphi_{\beta}^{-2}\left(u-u_{h}\right)\right\|_{W^{0, \beta}(\Omega)}=C\left\{\int_{\Omega} \varphi_{\beta}^{-2}\left|u-u_{h}\right|^{2} d x\right\}^{1 / 2} .
$$

By Lemma 2.4, if we define $\Xi=-\partial V / \partial n, x \in \partial \Omega$, then

$$
\|\Xi\|_{W^{1 / 2, \beta}(\partial \Omega)}<C\|V\|_{W^{2, \beta}(\Omega)} \text {. }
$$

By (2.5) and (5.24), we have $\Xi \in W^{1 / 2, \beta-\theta / 2}(\partial \Omega)$, so $\Xi \in W^{0, \beta / 2}(\partial \Omega)$. Noting also that, by Lemma 2.1,

$$
\int_{\Omega} \varphi_{\beta}^{-2}\left(u-u_{h}\right) v d x \leqslant C\left\|u-u_{h}\right\|_{H^{1}(\Omega)}\|v\|_{H^{1}(\Omega)}, \quad v \in H^{1}(\Omega),
$$

we see that the auxiliary problem admits the variational formulation

$$
(V, \Xi) \in H^{1}(\Omega) \times W^{0, \beta / 2}(\partial \Omega):
$$

$$
\mathscr{B}(v, \phi ; V, \Xi)=\int_{\Omega} \varphi_{\beta}^{-2}\left(u-u_{h}\right) v d x \quad \forall(v, \phi) \in H^{1}(\Omega) \times W^{0, \beta / 2}(\partial \Omega),
$$

where the bilinear form $\mathscr{B}$ is defined by (3.1).

By the same reasoning as above, we get, from (5.24) and (5.25), that

$$
\min _{(v, \phi) \in M_{\beta, k}^{h} \times N_{\beta, k}^{h}}\left\{\|(V-v, \Xi-\phi)\|_{\beta, h}^{2}\right\}<C h^{2} \int_{\Omega} \varphi_{\beta}^{-2}\left|u-u_{h}\right|^{2} d x .
$$

Then, since (1.4), (5.25), and (4.13) imply that

$$
\begin{aligned}
& \int_{\Omega} \varphi_{\beta}^{-2}\left|u-u_{h}\right|^{2} d x=\mathscr{B}\left(u-u_{h}, \psi-\psi_{h} ; V-v, \Xi-\phi\right) \\
& \quad \leqslant\left\|\left(u-u_{h}, \psi-\psi_{h}\right)\right\|_{\beta, h}\|(V-v, \Xi-\phi)\|_{\beta, h} \quad \forall(v, \phi) \in M_{\beta, k}^{h} \times N_{\beta, k}^{h},
\end{aligned}
$$


we obtain by combining (5.26) and (5.27) the estimate

$$
\int_{\Omega} \varphi_{\beta}^{-2}\left|u-u_{h}\right|^{2} d x<C h^{2}\left\|\left(u-u_{h}, \psi-\psi_{h}\right)\right\|_{\beta, h}^{2} .
$$

The asserted error bound now follows from the estimates (5.18) through (5.22), (5.28), and from Theorem 4.1.

Acknowledgment. The author would like to acknowledge a remark made by the referee, which led to an essential improvement in the proof of Lemma 4.1.

Institute of Mathematics

Helsinki University of Technology

SF-02150 Espoo 15, Finland

1. R. Adams, Sobolev Spaces, Academic Press, New York, 1975.

2. I. BABUŠKA, "The finite element method with Lagrange multipliers," Numer. Math., v. 20, 1973, pp. 179-192.

3. I. BABušKı \& A. K. AZIz, "Survey lectures on the mathematical foundations of the finite element method," The Mathematical Foundations of the Finite Element Method with Applications to Partial Differential Equations (A. K. Aziz, Ed.), Academic Press, New York, 1972.

4. I. Babušsa, R. B. Kellogg \& J. Prtkn̈ranta, "Direct and inverse error estimates for finite elements with mesh refinements," Numer. Math., v. 33, 1979, pp. 447-471.

5. J. BeroH \& J. LötsTröm, Interpolation Spaces, Springer-Verlag, Berlin, 1976.

6. F. BREZZI, "On the existence, uniqueness and approximations of saddle point problems arising from Lagrange multipliers," RAIRO Anal. Numér., v. 8, 1974, pp. 129-151.

7. P. G. Ciarlet, The Finite Element Method for Elliptic Problems, North-Holland, Amsterdam, 1978.

8. V. A. KonDRAT'Ev, "Boundary problems for elliptic equations in domains with conical or angular points," Trans. Moscow Math. Soc., v. 16, 1967, pp. 227-313.

9. J. Ptrkäranta, "Boundary subspaces for the finite element method with Lagrange multipliers," Numer. Math., v. 33, 1979, pp. 273-289.

10. J. Prträranta, "Local stability conditions for the Babuška method of Lagrange multipliers," Math. Comp., v. 35, 1980, pp. 1113-1129.

11. H. Triebel, "Interpolation theory for function spaces of Besov type defined in domains. II," Math. Nachr., v. 58, 1973, pp. 63-86. 